\title{
HRCT lung abnormalities are not a surrogate for exercise limitation in bronchiectasis
}

\author{
E.A. Edwards, I. Narang, A. Li, D.M. Hansell, M. Rosenthal, A. Bush
}

HRCT lung abnormalities are not a surrogate for exercise limitation in bronchiectasis. E.A. Edwards, I. Narang, A. Li, D.M. Hansell, M. Rosenthal, A. Bush. (C) ERS Journals Ltd 2004.

ABSTRACT: In paediatric bronchiectasis, there has been limited work on the relationship between disease severity as assessed by exercise limitation and high resolution computed tomography (HRCT).

An observational study was performed on 36 children who completed a questionnaire, physical examination, spirometry and sputum analysis, followed by a low dose of radiation protocol chest computed tomography (CT) scan and cycle ergometry incremental exercise test. A modified Bhalla score was used to score the HRCT abnormalities. The exercise variables used to assess functional status were heart rate, oxygen consumption, oxygen saturations and time of exercise. The results were compared with established normal paediatric values.

The median (range) age of the children was 13 yrs (10.6-17.1). Age, sex, height, weight or pubertal status were equally distributed between the children with cystic fibrosis (CF) or non-CF bronchiectasis. The children with non-CF bronchiectasis had a lower median forced expiratory volume in one second \% predicted than the children with $\mathrm{CF}(69 \%$ versus $76 \%$, respectively). The distribution of lung disease differed between the two groups. The children with CF bronchiectasis had predominantly right upper lobe disease, and scored higher for the presence and extent of bronchiectasis. Otherwise, there was no statistical difference in the overall CT score or the individual CT parameters between the groups. There was evidence of exercise limitation in both $\mathrm{CF}$ and non-CF bronchiectasis compared to normal children. No consistent relationships were identified between the lung function parameters, HRCT findings or exercise parameters in either disease group.

In this study, high resolution computed tomography features were not found to be markers of exercise capacity. As spirometry, high resolution computed tomography features and exercise testing give different information in children with cystic fibrosis and non-cystic fibrosis bronchiectasis, the current authors suggest all three may be required for the comprehensive assessment of respiratory status.

Eur Respir J 2004; 24: 538-544.
Depts of *Paediatric Respiratory Medicine and "Radiology, Royal Brompton Hospital, London, UK

Correspondence: A. Bush

Dept of Paediatric Respiratory Medicine

Royal Brompton Hospital

Sydney Street

London SW3 6NP

UK

Fax: 442073518754

E-mail: a.bush@rbh.nthames.nhs.uk

Keywords: Bronchiectasis

children

computed tomography scan

cystic fibrosis

exercise

Received: December 292003

Accepted after revision: May 172004

E.A. Edwards was supported by a fellowship awarded from the Health Research Council of New Zealand.
There is little consensus on the ideal follow-up or monitoring of disease progression in children with non-cystic fibrosis $(\mathrm{CF})$ bronchiectasis [1]. It has been suggested that the management should mirror the $\mathrm{CF}$ model $[2,3]$. There are at least two problems with this proposal. First, there is no gold standard for assessing disease severity or progression and, secondly, there are many differences between these two diseases. $\mathrm{CF}$ is a multisystem disease characterised by exaggerated airway inflammatory responses and infection with a relatively narrow spectrum, disease-specific group of respiratory pathogens, which only partially overlap with those in non-CF bronchiectasis. Non-CF bronchiectasis is a heterogeneous disease of varying severity and progression. The diagnosis may be reversible [4] and the decline in lung function with time may not be as steep as in CF [5-7]. There may be a different biofilm environment in the airways [8]. The distribution of lung disease in the two diseases has also been shown to vary $[9,10]$.

It has been known for a long time that standard spirometry is not a particularly sensitive measure of disease progression, but, by default, has become the marker to monitor progress. Some CF centres have moved towards a high resolution computed tomography (HRCT) chest scan every second year
[11] to quantify the progression of structural lung changes This is based on evidence that HRCT can detect early structural lung damage before lung function abnormalities are apparent [12-14]. However, there are no clinical data to allow a judgement on the benefit of the technique and no longitudinal studies in non-CF bronchiectasis. What limited crosssectional work there is on HRCT and non-CF bronchiectasis is retrospective and conflicting $[15,16]$.

In children, abnormalities on chest HRCT correlate negatively with lung function (forced expiratory volume in one second (FEV1), FEV1/forced vital capacity (FVC) and maximum expiratory flow at $25 \%$ of the FVC) [14, 17-19]. In adults with non-CF bronchiectasis, strong correlations have been demonstrated between the number of abnormal airways found on HRCT and the degree of impairment of pulmonary function [20]. This has lead to the suggestion that HRCT is useful to grade disease severity. A more recent longitudinal adult study demonstrated that the severity of bronchial wall thickness was the primary determinant of functional decline, implying that HRCT scans may be useful to monitor disease progression [21]. Nevertheless, it is unclear how complete a description of the status of a patient with bronchiectasis is provided by a HRCT scan. Although HRCT assesses 
structure and exercise function, and structure is one determinant of lung mechanics, a relationship between the two may be expected to exist. However, the strength of any such relationship is unknown. This is of practical importance, because if the relationship is very close there is no point in performing both tests as part of a detailed assessment of functional status, whereas if their relationship is loose, in other words if each test is giving different information, then the omission of one would result in the sacrifice of potentially useful information.

In some paediatric respiratory centres, the annual assessment of children with CF now includes some form of exercise study [22]. This has been based on evidence of significant limitation of aerobic and anaerobic exercise [23], and that this limitation has a relationship with survival [24]. There is little work that evaluates the exercise capability of children with non-CF bronchiectasis $[25,26]$ and there is only preliminary data in adults [27-30].

The current authors know of no study to date that examines the relationship between the severity of disease as measured by HRCT and exercise capability in CF and nonCF bronchiectasis. Therefore, it was hypothesised that there would be no relationship between the severity of lung disease as measured by HRCT and exercise limitation in either $\mathrm{CF}$ or non-CF bronchiectasis. A second hypothesis was that the functional severity of $\mathrm{CF}$ as judged by exercise testing would be greater than in non-CF bronchiectasis, reflecting the generally better prognosis. To investigate this, an observational study was performed to evaluate the severity of lung disease (assessed by a low-radiation-dose HRCT protocol) with functional status (assessed by a cycle ergometry exercise test) in children with $\mathrm{CF}$ and non-CF bronchiectasis, and compared the exercise parameters with previously established normal values.

\section{Methods}

\section{Subjects}

All patients regularly attended paediatric respiratory clinics at the Royal Brompton Hospital, London, UK. Bronchiectasis was defined by evidence of dilatation of the bronchial tree on HRCT scan performed at a time of stability [31] and confirmed with an appropriate clinical history of a chronic productive cough. This was according to the radiological criteria of NAIDICH et al. [32], which requires demonstration of a lack of normal bronchial tapering or any bronchi to have an internal diameter greater than the diameter of the accompanying pulmonary artery. $\mathrm{CF}$ and primary ciliary dyskinesia (PCD) were confirmed or excluded in all the children using standard criteria $[33,34]$. Any child who had a previous lung resection was excluded. No child was known to be an active smoker. All children were recruited at a time of disease stability and had not had an exacerbation for $\geqslant 1$ month.

The subjects were required to be $>8$ yrs old in order to be able to undertake the manoeuvres on the respiratory mass spectrometer (RMS) and have a height $>125 \mathrm{~cm}$ so that they could reach the pedals on the cycle ergometer. Exclusion criteria were premature birth, low birthweight $(<2.0 \mathrm{~kg})$, infection with Burkholderia cepacia, co-existing insulin-dependent diabetes mellitus, arthritis or arthralgia, and resting oxygen saturations $<90 \%$.

\section{Study design}

All subjects completed a questionnaire of relevant respiratory history and underwent a physical examination, followed by spirometry and sputum or cough swab culture. Puberty was scored using the Tanner scoring system [35]. The subjects then underwent a low-dose protocol chest computed tomography (CT) scan and a cycle ergometry incremental exercise test. The main outcome variables of the exercise test were heart rate (HR), oxygen consumption $\left(V^{\prime} \mathrm{O}_{2}\right)$, oxygen saturation $\left(\mathrm{Sa}, \mathrm{O}_{2}\right)$ and time of exercise. These results were then compared with established normal paediatric values [36]. All investigations were planned to be completed within a 1-month period.

The questionnaire determined the date of the last chest exacerbation, current medications, personal and family history of smoking, and the average number of hours of organised sport undertaken each week. Spirometry was performed using a compact portable spirometer (Vitalograph, Buckingham, UK). The FEV1, FVC and the forced expiratory flow between $25-75 \%$ of the FVC (FEF25-75) were recorded as percentage predicted for sex and height [37]. Sputum collection was attempted in every patient for microbiological assessment. A cough swab was performed where this was not possible. The results were then compared with the children's previous sputum results. Chronic sputum infection, defined as the repeated growth of one organism(s) on three or more occasions $\geqslant 1$ month apart over a 12-month period, was verified and recorded.

\section{High resolution computed tomography chest scan}

Inspiratory HRCT was performed using a Siemens Volume Zoom multi-slice CT scanner (Siemens, Erlangen, Germany) without sedation. Five 1-mm thick slices were taken, evenly interspaced between the level of the aortic arch and $1 \mathrm{~cm}$ above the right hemidiaphragm. This protocol, modified from that used in interstitial lung disease in adults, ensured that all lung segments were visualised and functionally significant disease was identified, while keeping radiation exposure to a minimum. The protocol varied the milliampere-seconds according to the weight of the child. The acquisition for each section was $0.75 \mathrm{~s}$. The total effective dose to the child was $\leqslant 0.1 \mathrm{mSv}$, a factor of 10 less than the standard radiation dose for a CT chest of a child. The inspiratory scans were then scored by one radiologist (D.M. Hansell) using a modification of the Bhalla score previously used in children and adults with $\mathrm{CF}$ and non-CF bronchiectasis [16, 19, 20, 38]. Using this system, each lung was evaluated according to the individual lobes, with the lingula being considered separately. Features recorded included the presence of bronchiectasis, the degree of bronchial wall thickening and bronchial dilatation, and the presence of mucous plugging, air-trapping, atelectasis or consolidation. The CT score of each child was taken as the sum of each of the lobe scores. The radiologist was blinded to the diagnoses of the children.

\section{Exercise test}

The ambient pressure, temperature and per cent humidity were recorded and entered into the RMS. The ambient temperature was never $>26^{\circ} \mathrm{C}$. During manoeuvres, the children had continuous pulse rate and arterial $\mathrm{Sa}, \mathrm{O}_{2}$ measured using a surface oximeter (Nellcor, Hatwood, CA, USA) placed over the right supraorbital artery and fixed with a bandanna, this site being the least prone to exercise artefact. Detailed descriptions of the protocols have been described elsewhere [39]. Briefly, the subject exercised using an electromagnetic bicycle (Seca 100, Birmingham, UK), which produced a constant workload independent of pedal speeds over the 
range of 6-150 revolutions $\cdot \mathrm{min}^{-1}$. The subject started exercising by initially cycling backwards at zero load to warm up for $1 \mathrm{~min}$ and then forwards, initially at $25 \mathrm{~W} \cdot \mathrm{m}^{-2}$, increasing in $15-\mathrm{W} \cdot \mathrm{m}^{-2}$ increments every $3 \mathrm{~min}$ until exhaustion, heralded by rocking and/or progressive speeding up and slowing down on the cycle, as in previous reports [36]. The pulse rate was almost always $>190 \cdot \mathrm{min}^{-1}$ and, at the end of the protocol, no child stated that they could have undertaken an additional 3min stage. For consistency, children were encouraged to pedal at 50-70 rpm during the entire duration of the exercise.

\section{Respiratory mass spectrometry}

An Innovision 2000 quadrupole mass spectrometer (Odense, Denmark) was used to sample subjects' ventilated gas and analyse it on the basis of its mass:charge ratio to determine $V^{\prime} \mathrm{O}_{2}$. The hardware and physiological measurements are fully described elsewhere [39, 40].

\section{Data analysis}

For the purposes of this paper, only the curves related to $V^{\prime} \mathrm{O}_{2}$ during exercise, as measured by the mass spectrometer, were analysed. The children's clinical characteristics, HRCT scores and pulmonary function indices were expressed as mean \pm SD or median (range), depending upon whether they were normally distributed. Values obtained from normal children and their SD scores [39, 40] were used to obtain mean and $95 \%$ confidence interval z-scores for the children and for the different variables. The $z$-scores were adjusted for age, height, weight (and, hence, surface area defined as the square root of (height $\times$ weight)/3,600) and puberty. When the distribution of variables was normal, paired t-tests were used; when the distribution was not normal, Mann-Whitney (rank sum) tests were performed to compare the differences between disease groups. Analysis of correlations between the exercise variables, HRCT scores and other variables was performed using Spearman's rank correlation coefficient $(\rho)$.

\section{Ethics}

The local ethics committee gave approval for this study. Written informed consent was obtained from all parents of the children taking part in this study, along with assent from the children themselves.

\section{Results}

\section{Subjects}

A total of 40 children were originally approached. Despite previous HRCT evidence of bronchiectasis, four children were excluded. One child had a previous lobectomy and three did not have clinically significant disease on the repeat HRCT. Thus, data were analysed on the remaining 36 children, of whom 18 had bronchiectasis due to CF and 18 had bronchiectasis due to other causes. Of these, six children had bronchiectasis as a result of PCD, one child had bronchiectasis attributed to previous pertussis and another as a result of tracheo-oesophageal fistula and severe gastro-oesophageal reflux. In the remaining children $(n=9)$, despite intensive investigation (including sweat test, $\mathrm{CF}$ genotyping, ciliary structure and function, immune screen, and $\mathrm{pH}$ study), no underlying cause for the bronchiectasis had been found. The demographic features and lung function parameters of the children are shown in table 1.

The median (range) age for both groups of children was 13.0 yrs (10.6-17.1), and there was no difference in the age, sex, height, weight or pubertal status $(\mathrm{p}>0.05)$. There was no statistical difference in FVC or FEF25-75, but there was a difference in the median FEV1 between the two groups $(\mathrm{CF}$ FEV1 76\% (40-106) versus non-CF bronchiectasis FEV1 69\% $(35-90) ; \mathrm{p}=0.03)$. In total, $10(56 \%)$ of the children with $\mathrm{CF}$ were $\Delta$ F508 homozygous, $14(78 \%)$ were chronically infected with Pseudomonas aeruginosa and $16(89 \%)$ were pancreatic insufficient. Three of the children with bronchiectasis were chronically infected with Haemophilus influenzae, but none had ever grown $P$. aeruginosa. None of the children with non$\mathrm{CF}$ bronchiectasis demonstrated digital clubbing, whereas five $(28 \%)$ of the children with CF did. Only two cases in each group showed chronic chest deformity. None of the children with CF had wheeze on auscultation, but two had crackles. Two of the children with non-CF bronchiectasis had wheeze and seven had crackles.

A total of $12(67 \%)$ of the children with CF were prescribed inhaled corticosteroids (dose $800 \mu \mathrm{g}(400-2000))$ and bronchodilators. Fewer $(n=9,50 \%)$ children with non-CF bronchiectasis were prescribed inhaled corticosteroids (dose $500 \mu \mathrm{g}$ (200-800)), but the difference did not reach significance $(\mathrm{p}=0.58) ; 11$ were prescribed bronchodilators. Eight and four were prescribed long-acting bronchodilators in the $\mathrm{CF}$ and non-CF groups, respectively. The 14 children with $P$. aeruginosa were all prescribed nebulised antibiotics (either gentamicin and colomycin, or colomycin alone). In total, 12 of the children with $\mathrm{CF}$ were on long-term oral antibiotics (nine were prescribed flucloxacillin and three were prescribed azithromycin), whereas only six of the children with non-CF bronchiectasis were prescribed prophylactic oral antibiotics (two were prescribed cefaclor and four were prescribed amoxycillin/clavulanic acid; $\mathrm{p}=0.004)$.

All the study tests were completed on the same day in 30 out of 36 children; the remaining six children completed the tests within 10 days.

\section{High resolution computed tomography scans}

The children with CF had more widespread disease (lobes 6 (3-6) versus non-CF bronchiectasis $4.5(1-6) ; p=0.02)$. The distribution of disease was different between the two groups (fig. 1). The worst affected lobe in the children with CF was the right upper lobe $(\mathrm{p}=0.002)$ and the non-CF group had predominantly lower lobe disease, but the difference did not reach significance (right lower lobe $\mathrm{p}=0.65$, left lower lobe $\mathrm{p}=0.58$ ). Except for the presence and extent of bronchiectasis

Table 1.-Demographic features of the children together with the lung function parameters of the children with cystic fibrosis (CF) and non-CF bronchiectasis

\begin{tabular}{lccc}
\hline Demographics & Normals & CF & Bronchiectasis \\
\hline Subjects (females) & $106(51)$ & $18(11)$ & $18(7)$ \\
Age yrs & $12.5(8.0-16.9)$ & $13.0(10.7-17.0)$ & $13.0(10.6-17.1)$ \\
Exercise $\cdot$ week $^{-1} \mathrm{~h}$ & & $4.5(1.5-12)$ & $3.5(1-16)$ \\
Weight kg & $46(23-75)$ & $47(31-67)$ & $46(33-65)$ \\
Height cm & $154(129-183)$ & $154(140-170)$ & $159(137-183)$ \\
FVC \% pred & & $93(47-113)$ & $84(55-115)$ \\
FEV1\% pred & & $76(40-106)$ & $69(35-90)^{\#}$ \\
FEF25-75\% pred & & $50(25-104)$ & $39(13-97)$ \\
\hline
\end{tabular}

Data are presented as $\mathrm{n}$ and median (range). FVC: forced vital capacity; FEV1: forced expiratory volume in one second; FEF25-75: forced midexpiratory flow. ${ }^{\#}$ : $\mathrm{p}=0.03$. 


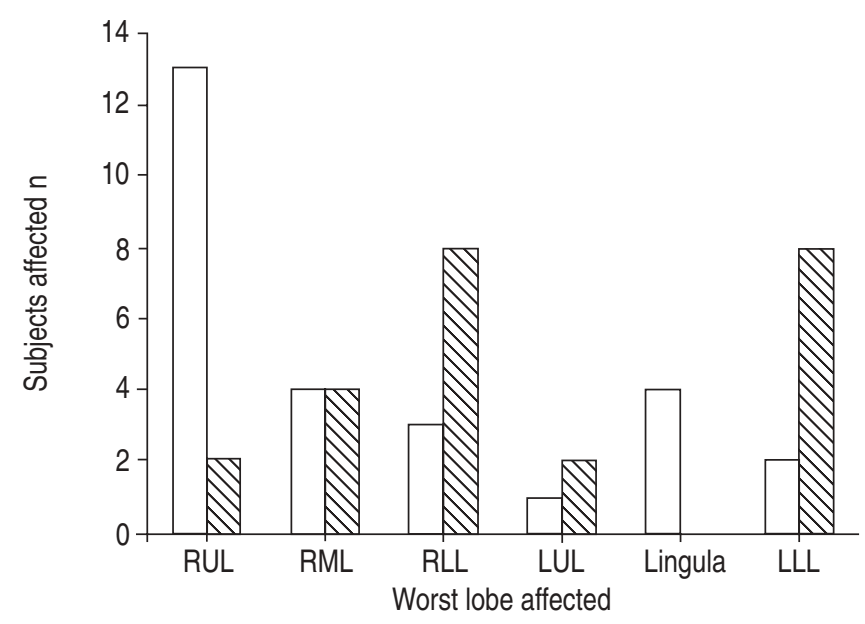

Fig. 1.- The worst lung lobes affected by bronchiectasis in the children with cystic fibrosis $(\mathrm{CF} ; \square)$ and non-CF bronchiectasis $(\mathbb{\mathbb { Q }})$ as defined by the modified Bhalla score. The children with CF had predominately right upper lobe (RUL) disease $(\mathrm{p}=0.002)$, while the children with non-CF bronchiectasis had lower lobe disease. Five children had $>1$ lobe that was given the same score, six children with bronchiectasis had $>1$ lobe with the same score. RML: right middle lobe; RLL: right lower lobe; LUL: left upper lobe; LLL: left lower lobe.

$(\mathrm{p}=0.001)$, there was no significant difference in the median total CT scores or the individual CT parameters as assessed by the modified Bhalla score for the children with $\mathrm{CF}$ and non-CF bronchiectasis (table 2).

There were no consistent correlations between lung function, clinical parameters (self-reported hours of exercise per week, CF genotype and chronic infection) or physical findings (digital clubbing and chest deformity) for either the children with or without $\mathrm{CF}$. In the children with non-CF bronchiectasis, only air trapping demonstrated a relationship with the median FEV1 and median FEF25-75 ( $\mathrm{r}=-0.41$ and $\mathrm{r}=-0.49$, respectively). Only the presence and extent of bronchiectasis related weakly to FEV1 in the CF group ( $r=-0.38)$. None of the other CT parameters or the total CT score had any significant relationships with lung function in the children with $\mathrm{CF}$ or non-CF bronchiectasis. When the total scores for

Table 2. - Total computed tomography (CT) scores for the individual lung lobes and the individual $\mathrm{CT}$ features for the children with cystic fibrosis (CF) and non-CF bronchiectasis

$\mathrm{CF} \quad$ Bronchiectasis

\begin{tabular}{lcc}
\hline Lung lobes affected & & \\
RUL & $5(3-9)$ & $3(0-7)^{\#}$ \\
RML & $5(0-7)$ & $4(0-9)$ \\
RLL & $4(0-8)$ & $6(0-7)$ \\
LUL & $4(0-7)$ & $3(0-6)$ \\
Lingula & $5(0-7)$ & $4(0-8)$ \\
LLL & $4(1-7)$ & $6(0-8)$ \\
CT scores & & \\
Bronchiectasis & $11(2-15)$ & $6(1-12)^{* * *}$ \\
Bronchial wall dilatation & $6(1-8)$ & $5(1-8)$ \\
Bronchial wall thickness & $1(0-7)$ & $4(1-6)$ \\
Mucus plugging & $0(0-3)$ & $0(0-5)$ \\
Air trapping & $6(0-13)$ & $3(0-13)$ \\
Atelectasis consolidation & $0(0-1)$ & $0(0-2)$ \\
Total CT score & $27(6-44)$ & $18(3-39)$ \\
\hline
\end{tabular}

Data are presented as median (range). RUL: right upper lobe; RML: right middle lobe; RLL: right lower lobe; LUL: left upper lobe; LLL: left lower lobe. ${ }^{\#}: \mathrm{p}=0.002 ; * * *: \mathrm{p}=0.001$. the individual lung lobes most severely affected in each disease group were compared with lung function and clinical data, there were still no consistent relationships in the children with $\mathrm{CF}$. The children with non-CF bronchiectasis demonstrated moderate relationships between the right lower lobe and the right upper lobe and lung function (right lower lobe: FEV1 r=-0.49, FEF25-75 r=-0.47; right upper lobe: FEV1 $\mathrm{r}=-0.41, \mathrm{FEF} 25-75 \mathrm{r}=-0.49)$.

\section{Exercise test}

Only the curves related to $V^{\prime} \mathrm{O}_{2}$ during exercise were analysed. There was no difference in the median time of exercise between the two groups ( $\mathrm{CF} 9 \mathrm{~min}(6-15)$ and non-CF bronchiectasis $9 \mathrm{~min}(6-15)$ ). The resting $\mathrm{Sa}, \mathrm{O}_{2}$ in the $\mathrm{CF}$ group was $99 \%(97-100)$ and in the non-CF bronchiectasis group it was also $99 \%(97-100)$. The $\mathrm{Sa}_{2} \mathrm{O}_{2}$ at the end of exercise in the $\mathrm{CF}$ group was $99 \%(97-100)$ and in the non-CF bronchiectasis group was $98 \%(96-100)$. The change in $\mathrm{Sa}_{2} \mathrm{O}_{2}$ after exercise was $0 \%$ in the $\mathrm{CF}$ group and $\pm 1 \%$ in the bronchiectasis group. There were no significant desaturations (defined as change in $\mathrm{Sa}, \mathrm{O}_{2}>4 \%$ ) in either group, and there were no differences between $\mathrm{CF}$ and non-CF bronchiectasis $(\mathrm{p}>0.05)$.

$\mathrm{HR}$ and $V^{\prime} \mathrm{O}_{2}$ are represented as z-scores compared with normal children who had previously undergone exercise testing using an identical protocol in the current authors' institution. These are represented in table 3. Full details of the control children and their results have been reported elsewhere [36]. The z-scores for HR at rest and each exercise stage for both groups were significantly greater when compared to healthy children $(\mathrm{p}<0.05)$. There was no difference between $\mathrm{CF}$ and non-CF bronchiectasis $(\mathrm{p}>0.05)$. The mean HR was $\sim 16$ beats $\cdot \mathrm{min}^{-1}$ higher after $9 \mathrm{~min}$ of exercise in the disease groups compared to controls. The z-scores for $V^{\prime} \mathrm{O}_{2}$ (fig. 2) showed that, in the $\mathrm{CF}$ group, at rest and any given exercise stage, $V^{\prime} \mathrm{O}_{2}$ was greater than in healthy children $(\mathrm{p}<0.05)$. For the non-CF bronchiectasis group, the $V^{\prime} \mathrm{O}_{2}$ was normal at rest and exercise stage 1 , but was greater than in normal children at exercise stage 2 and $3(p<0.05)$. There was no difference between $\mathrm{CF}$ and non- $\mathrm{CF}$ bronchiectasis $(\mathrm{p}>0.05)$ at rest and any exercise stage. At exercise stage 3 , the mean increase in $V^{\prime} \mathrm{O}_{2}$ was in the order of $200 \mathrm{~mL} \cdot \mathrm{min}^{-1} \cdot \mathrm{m}^{-2}$ for both groups.

There was no relationship between any of these measured

Table 3.-Z-scores for heart rate (HR) and oxygen consumption $\left(\mathrm{V}^{\prime} \mathrm{O}_{2}\right)$ at the different exercise stages for the children with cystic fibrosis (CF) and non-CF bronchiectasis

\begin{tabular}{lll}
\hline $\begin{array}{l}\text { Exercise stage } \\
\text { of measured } \\
\text { variable }\end{array}$ & $\mathrm{CF}$ & Bronchiectasis \\
\hline zHR & & \\
Rest & $1.15^{*}(0.67-1.63)$ & $0.90^{*}(0.46-1.34)$ \\
Stage 1 & $1.41^{*}(0.84-1.98)$ & $1.29^{*}(0.67-1.90)$ \\
Stage 2 & $0.92^{*}(0.55-1.28)$ & $1.12^{*}(0.53-1.72)$ \\
Stage 3 & $0.86^{*}(0.41-1.32)$ & $0.91^{*}(0.18-1.64)$ \\
z $V^{\prime} \mathrm{O}_{2}$ & & \\
Rest & $0.48^{*}(0.12-0.85)$ & $0.59(-0.13-1.33)$ \\
Stage 1 & $0.51^{*}(0.06-0.96)$ & $0.42(-0.15-0.99)$ \\
Stage 2 & $1.35^{*}(0.56-2.14)$ & $0.86^{*}(0.23-1.48)$ \\
Stage 3 & $1.40^{*}(0.60-2.20)$ & $0.95^{*}(0.02-1.87)$ \\
\hline
\end{tabular}

Data are presented as median (range). There was no significant difference between the two disease groups for either parameter at rest or at any exercise stage. $*$ : $\mathrm{p}<0.05$ for that measurement compared to corresponding value for the normal controls. 


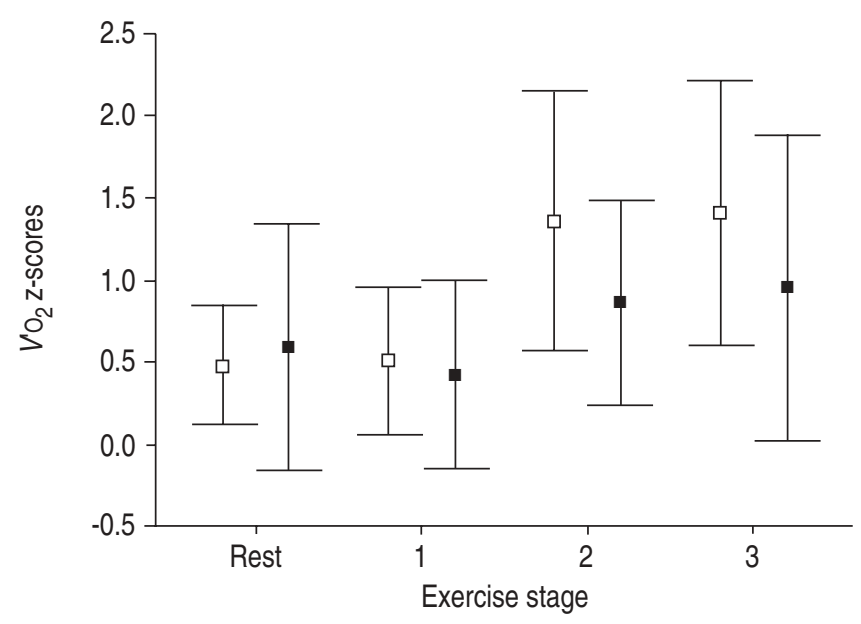

Fig. 2.-Z-scores for oxygen consumption $\left(V^{\prime} \mathrm{O}_{2}\right)$ in the children with cystic fibrosis (CF; $\square$ ) and non-CF bronchiectasis ( $\square$ ). Z-scores are significantly elevated at exercise stages 2 and 3 for both groups (error bars do not overlap zero). In the $\mathrm{CF}$ group, at rest and at any given exercise stage, $V^{\prime} \mathrm{O}_{2}$ was greater than in healthy children $(\mathrm{p}<0.05)$. For the non-CF bronchiectasis group, the $V^{\prime} \mathrm{O}_{2}$ was normal at rest and exercise stage 1 , but was greater than in normal children at exercise stages 2 and $3(\mathrm{p}<0.05)$. In $\mathrm{CF}$ children, $\mathrm{n}=18,18,18$ and 14 at rest, stage 1,2 and 3 , respectively. In non-CF bronchiectasis, $n=18,18,18$ and 12 at rest, stage 1,2 and 3 , respectively.

variables and lung function, chronic infection with $P$. aeruginosa, use of inhaled corticosteroids, antibiotic therapy and self-reported levels of activity. There were no consistent correlations between any of the outcome variables of the exercise test and the structural parameters of lung disease as assessed by HRCT in either disease (table 4).

\section{Discussion}

This limited study suggests that in $\mathrm{CF}$ and non-CF bronchiectasis HRCT chest features are not a useful marker of functional status, as judged by a cycle ergometer exercise test. This was despite evidence of exercise limitation and abnormal HRCTs in both $\mathrm{CF}$ and non-CF bronchiectasis in comparison with normal children. No consistent relationships were identified between the lung function and HRCT scan

Table 4.-Univariate (Spearman $\rho$ ) correlations between structural computed tomography (CT) features and exercise parameters measured

\begin{tabular}{lcccr}
\hline CT features & Disease & \multicolumn{3}{c}{ Exercise parameters } \\
\cline { 3 - 5 } & & $\mathrm{Sa}_{\mathrm{a}, \mathrm{O}_{2}}$ & $\mathrm{z} V^{\prime} \mathrm{O}_{2}$ & $\mathrm{zHR}$ \\
\hline Bronchiectasis & Bronchiectasis & -0.05 & -0.23 & -0.15 \\
& $\mathrm{CF}$ & -0.14 & 0.09 & -0.01 \\
Wall dilatation & Bronchiectasis & -0.11 & -0.15 & -0.14 \\
& $\mathrm{CF}$ & -0.17 & 0.16 & 0.08 \\
Wall thickness & Bronchiectasis & -0.22 & -0.11 & -0.13 \\
& $\mathrm{CF}$ & $0.40^{\#, \oplus}$ & $<0.01$ & 0.03 \\
Air trapping & Bronchiectasis & -0.12 & -0.11 & 0.02 \\
& $\mathrm{CF}$ & -0.19 & $0.33^{\#,+}$ & 0.04 \\
Total CT score & Bronchiectasis & -0.05 & -0.25 & -0.12 \\
& $\mathrm{CF}$ & -0.06 & 0.21 & 0.13 \\
& & & &
\end{tabular}

Data are presented as n. CF: cystic fibrosis; $\mathrm{Sa}, \mathrm{O}_{2}$ : oxygen saturation; $V^{\prime} \mathrm{O}_{2}$ : oxygen consumption; HR: heart rate. $\mathrm{p}$-Values are given where weak relationships were demonstrated. ${ }^{\#}$ : r-values; ${ }^{\uparrow}: \mathrm{p}=0.0001{ }^{+}$: $\mathrm{p}=0.006$. findings in $\mathrm{CF}$ and non-CF bronchiectasis. The HRCT scores were similar. Despite the perception of non-CF bronchiectasis as having a better prognosis than $\mathrm{CF}$, the degree of exercise limitation was the same in the two groups. The main difference in CT findings was that the distribution of lung disease in the children with $\mathrm{CF}$ bronchiectasis mainly affected the right upper lobe, whereas, in the children with non-CF bronchiectasis, the lower zones were predominantly affected. This is in keeping with previously published work $[9,10]$.

The main strengths of this prospective study are that the majority of the children had their exercise test on the same day as their HRCT, and the CT score and the exercise tests were all performed by the same experienced investigators (D.M. Hansell and I. Narang, respectively). The main limitations, however, are the relatively small sample size and, also, the heterogeneity of diagnoses in the non-CF bronchiectasis group. The small sample size does mean that statistically significant $p$-values should be interpreted with caution. It should be noted that the groups are comparable to other exercise studies in this field $[25,26]$. However, examination of the scatter of the data suggests that the current conclusions are likely to be broadly correct, although ideally requiring confirmation from another larger study. A further issue is that the range of $V^{\prime} \mathrm{O}_{2}$ values in healthy children is wide, which compounds the difficulty in using such a measure as a predictor of severity in disease. With regard to sample size, there was insufficient specific information in this area to perform power calculations prior to commencing the study. However, the data generated by this study could be used to perform power calculations using the mean $V^{\prime} \mathrm{O}_{2}$ deviation at exercise stage 3 . If the $V^{\prime} \mathrm{O}_{2}$ is $200 \pm 50 \mathrm{~mL}$, a difference of $100 \mathrm{~mL}$ between the bronchiectatic groups requires seven subjects in each group, a difference of $50 \mathrm{~mL}$ requires 20 in each group and a difference of only $25 \mathrm{~mL}$ requires 60 in each group, in order to be detected with $85 \%$ certainty at the $5 \%$ level. The current authors feel that a difference of $50 \mathrm{~mL}$ is the minimum relevant difference; therefore, 18 subjects in each group should be sufficient. Taking into account the inclusion and exclusion criteria, more than this number would have been difficult to achieve even in a large tertiary paediatric respiratory centre. Given the size and nature of the study groups, the findings described in this study may not be applicable to all subgroups of non-CF bronchiectasis. However, it is believed that the findings reported in this study do give justification for a larger multicentre prospective study, including data on which to base future power calculations.

Although one expert radiologist scored the CT scans, the CT protocol was validated in adults with interstitial lung disease, and an assessment of reproducibility would have added strength to the protocol. The limited correlations with lung function are in keeping with the work of CHANG et al. [15], but are in contrast to other work utilising the modified Bhalla score [16, 19-21]. There are a number of possible explanations for this. Disease may have been missed by performing only five slices. However, bronchiectasis is not a skip lesion and the position of the slices should have included all of the lung segments. It seems unlikely that clinically significant disease was missed.

The children used in this study appeared to represent a spectrum of clinical severity, but many were at the milder end of the disease spectrum. The slightly stronger correlations with lung function in the children with non-CF bronchiectasis may have been a consequence of worse lung function (FEV1) or wider span. While the latter is more likely, it highlights the importance of caution in extrapolating relationships across different disease groups. The children were in the second decade of life and, because of this and the cross-sectional design, the effect of age on CT and exercise outcomes could not be assessed. The age and ability to do the exercise test not 
only limited the sample size in this study, but also raised a more general problem: cycle ergometry utilising mass spectrometry is not a simple or readily available exercise test. Although it is a very good test to ascertain the ventilatory and haemodynamic responses that occur during exercise, it cannot be assumed that the abnormalities demonstrated in this study will be reproduced using more simple exercise methodology [41]. However, a mass spectrometer is not required to measure $V^{\prime} \mathrm{O}_{2}$.

Although historically the serial evaluation of chest radiographs has been reported as being suitable to document progression of CF [42] and non-CF bronchiectasis [43], it has been well established that HRCT of the chest provides more detailed information [44 46]. Utilising HRCT scans and a validated CT score in this study is a major strength in comparison with other work that uses chest radiograph assessment to compare with exercise.

COATES et al. [47] studied 18 subjects with CF (age 12 yrs (5-16)) and found that the degree of airways obstruction (as gauged by spirometry using maximum mid-expiratory flow (MMEF) rate), the exercise tolerance on a cycle ergometer and the clinical grading each correlated significantly with the chest radiograph score defined by HODSON and FRANCE [48] (chest radiograph and clinical score $r=0.73$, MMEF $r=0.72$ and maximum work as \% pred $\mathrm{r}=0.80$ [48]). AKALIN et al. [25] attempted to evaluate the cardiac effects of non-CF bronchiectasis by comparing a treadmill exercise test with novel clinical and chest radiograph scores, pulmonary function, and a resting echocardiogram. With the exception of bronchodilator therapy, no comment was made on current medication (particularly steroids and antibiotics) or chronic sputum infection. The study also reported that $43 \%$ of the 21 children had previous surgery for bronchiectasis, which was an exclusion criterion in the current work. The high surgery rates $(40 \%)$ and high prevalence of $P$. aeruginosa chronic infection $(29 \%)$, in contrast to the current non-CF cohort, has been reported in earlier papers on non-CF bronchiectasis in Turkey $[49,50]$. There was no comment on the extent of lobar resections and the effect this may have not only on exercise capability but also on how this was accounted for in the novel chest radiograph score. Finally, SwAminathan et al. [26] demonstrated impaired pulmonary function and exercise tolerance in children with non-CF bronchiectasis compared to normal controls, but they did not have data on imaging and were not able to compare their findings to a CF control group. There are currently no other studies known that evaluate the exercise capability of children with non-CF bronchiectasis.

This study was not designed to test whether the observations of exercise limitation or HRCT features are sensitive to changes with time or with interventions. No consistent relationships were found between HRCT features and pulmonary function; however, this may be the result of the children in the current study having mild disease. CT provides structural information, but not functional information in this group; therefore, both need to be performed for an assessment. The best prognostic guide or treatment cannot be determined from this study. Perhaps, as has been suggested in CF lung disease [51], the gold standard prognostic indicator of the future may not be pulmonary function or pulmonary function together with structural CT changes, but a more "holistic" (functional, anatomical and pathophysiological) evaluation by combining measurements of pulmonary function, structural CT changes and exercise capability. This remains to be tested in prospective studies. The longitudinal implications and possible dangers of exposure to cumulative amounts of even low-dose radiation have additional important ramifications on the management of paediatric respiratory disease.
In summary, this study has shown that basic cardiorespiratory parameters are compromised to an equal extent in cystic fibrosis and non-cystic fibrosis bronchiectasis. Whether exercise testing along the lines of the cystic fibrosis guidelines is appropriate or valuable for the assessment of non-cystic fibrosis bronchiectasis needs further clarification. In contrast to previous work, no consistent relationships were identified between lung function parameters and high resolution computed tomography scan findings, and may have been due to a milder spectrum of disease in the children examined. Spirometry, high resolution computed tomography and exercise testing give different information in children with cystic fibrosis and non-cystic fibrosis bronchiectasis. Pending justification by larger prospective longitudinal studies, it is suggested that all three could be combined in a composite score for the comprehensive assessment of respiratory status.

\footnotetext{
Acknowledgements. The authors would like to thank all the children and young people who took part in this study. The authors also thank S. Evans (Radiation Protection Adviser; Royal Marsden Hospital, London, UK) for his help with the chest computed tomography scan protocol.
}

\section{References}

1. Callahan CW, Redding GJ. Bronchiectasis in children: orphan disease or persistent problem? Pediatr Pulmonol 2002; 33: 492-496.

2. Chang AB, Grimwood K, Mulholland EK, Torzillo PJ. Bronchiectasis in indigenous children in remote Australian communities. Med J Aust 2002; 177: 200-204.

3. Cystic Fibrosis Trust, British Paediatric Association, British Thoracic Society. Clinical guidelines for cystic fibrosis care. London, Royal College of Physicians, 1996.

4. Gaillard EA, Carty H, Heaf D, Smyth RL. Reversible bronchial dilatation in children: comparison of serial highresolution computer tomography scans of the lungs. Eur J Radiol 2001; 47: 215-220.

5. Ellerman A, Bisgaard H. Longitudinal study of lung function in a cohort of primary ciliary dyskinesia. Eur Respir J 1997; 10: 2376-2379.

6. Noone PG, Leigh MW, Sannuti A, et al. Primary ciliary dyskinesia: diagnostic and phenotypic features. Am J Respir Crit Care Med 2004; 169: 459-467.

7. Barker AF, Couch L, Fiel SB, et al. Tobramycin solution for inhalation reduces sputum Pseudomonas aeruginosa density in bronchiectasis. Am J Respir Crit Care Med 2000; 162: 481485.

8. Prince AS. Biofilms, antimicrobial resistance, and airway infection. N Engl J Med 2002; 347: 1110-1111.

9. Gurney JW, Habbe TG, Hicklin J. Distribution of disease in cystic fibrosis: correlation with pulmonary function. Chest 1997; 112: 357-362.

10. Maffessanti M, Candusso M, Brizzi F, Piovesana F. Cystic fibrosis in children: HRCT findings and distribution of disease. $J$ Thorac Imaging 1996; 11: 27-38.

11. Tiddens HA. Detecting early structural lung damage in cystic fibrosis. Pediatr Pulmonol 2002; 34: 228-231.

12. de Jong PA, Nakano Y, Lequin MH, et al. Progressive damage on high resolution computed tomography despite stable lung function in cystic fibrosis. Eur Respir $J$ 2004; 23 : 93-97.

13. Long FR, Williams RS, Castile RG. Structural airway abnormalities in infants and young children with cystic fibrosis. J Pediatr 2004; 144: 154-161.

14. Marchant JM, Masel JP, Dickinson FL, Masters IB, Chang AB. Application of chest high-resolution computer 
tomography in young children with cystic fibrosis. Pediatr Pulmonol 2001; 31: 24-29.

15. Chang AB, Masel JP, Boyce NC, Wheaton G, Torzillo PJ. Non-CF bronchiectasis: clinical and HRCT evaluation. Pediatr Pulmonol 2003; 35: 477-483.

16. Edwards EA, Metcalfe R, Milne DG, Thompson J, Byrnes CA. A retrospective review of children presenting with noncystic fibrosis bronchiectasis: HRCT features and clinical relationships. Pediatr Pulmonol 2003; 36: 87-93.

17. Helbich TH, Heinz-Peer G, Eichler I, et al. Cystic fibrosis: CT assessment of lung involvement in children and adults. Radiology 1999; 213: 537-544.

18. Demirkazik FB, Ariyurek OM, Ozcelik U, Gocmen A, Hassanabad HK, Kiper N. High resolution CT in children with cystic fibrosis: correlation with pulmonary functions and radiographic scores. Eur J Radiol 2001; 37: 54-59.

19. Dakin CJ, Pereira JK, Henry RL, Wang H, Morton JR. Relationship between sputum inflammatory markers, lung function, and lung pathology on high-resolution computed tomography in children with cystic fibrosis. Pediatr Pulmonol 2002; 33: 475-482.

20. Roberts HR, Wells AU, Milne DG, et al. Airflow obstruction in bronchiectasis: correlation between computed tomography features and pulmonary function tests. Thorax 2000; 55: 198-204.

21. Sheehan RE, Wells AU, Copley SJ, et al. A comparison of serial computed and functional change in bronchiectasis. Eur Respir J 2002; 20: 1-7.

22. Orenstein DM. Exercise testing in cystic fibrosis. Pediatr Pulmonol 1998; 25: 223-225.

23. Shah AR, Gozal D, Keens TG. Determinants of aerobic and anaerobic exercise performance in cystic fibrosis. Am J Respir Crit Care Med 1998; 157: 1145-1150.

24. Nixon PA, Orenstein DM, Kelsey SF, Doershuk CF. The prognostic value of exercise testing in patients with cystic fibrosis. N Engl J Med 1992; 327: 1785-1788.

25. Akalin F, Koroglu TF, Bakac S, Dagli E. Effects of childhood bronchiectasis on cardiac functions. Pediatr Int 2003; 45: 169-174.

26. Swaminathan S, Kuppurao KV, Somu N, Vijayan VK. Reduced exercise capacity in non-cystic fibrosis bronchiectasis. Indian J Pediatr 2003; 70: 553-556.

27. O'Leary CJ, Wilson CB, Hansell DM, Cole PJ, Wilson R, Jones PW. Relationship between psychological well-being and lung health status in patients with bronchiectasis. Respir Med 2002; 96: 686-692.

28. Edmunds J, Moseley B, Sharples D, et al. Lung function and exercise tolerance in bronchiectasis: is small airways disease an important component in bronchiectasis. Am J Respir Crit Care Med 2001; 163: A715.

29. Koulouris NG, Retsou S, Kosmas EN, et al. Tidal expiratory flow limitation, dyspnoea and exercise capacity in patients with bilateral bronchiectasis. Eur Respir $J$ 2003; 21: 743-748.

30. Ozerovitch LJ, Shoemark AN, Jones P, Wilson R. Effects of extent of disease, FEV1 and exercise capacity on quality of life in bronchiectasis. Am J Respir Crit Care Med 2003; 167 : A746.

31. Hansell DM. Bronchiectasis. Radiol Clin North Am 1998; 36 : $107-128$.

32. Naidich DP, McCauley DI, Khouri NF, Stitik FP,
Siegelman SS. Computed tomography of bronchiectasis. J Comput Assist Tomogr 1982; 6: 437-444.

33. Rosenstein BJ, Cutting GR. The diagnosis of cystic fibrosis: a consensus statement. Cystic Fibrosis Foundation Consensus Panel. J Pediatr 1998; 132: 589-595.

34. Bush A, Cole P, Hariri M, et al. Primary ciliary dyskinesia: diagnosis and standards of care. Eur Respir J 1998; 12: 982 988.

35. Tanner JM. Growth at adolescence. Oxford, Blackwell Scientific Publications, 1962; pp. 28-39.

36. Rosenthal M, Bush A. Haemodynamics in children during rest and exercise: methods and normal values. Eur Respir $J$ 1998; 11: 854-865.

37. American Thoracic Society. Standardization of spirometry, 1994 update. Am J Respir Crit Care Med 1995; 152: 11071136.

38. Bhalla M, Turcios N, Aponte V, et al. Cystic fibrosis: scoring system with thin-section CT. Radiology 1991; 179: 783-788.

39. Rosenthal M, Redington A, Bush A. Cardiopulmonary physiology after surgical closure of asymptomatic secundum atrial septal defects in childhood. Exercise performance is unaffected by age at repair. Eur Heart J 1997; 18: 1816-1822.

40. Rosenthal M, Bush A, Deanfield J, Redington A. Comparison of cardiopulmonary adaptation during exercise in children after the atriopulmonary and total cavopulmonary connection Fontan procedures. Circulation 1995; 91: 372378.

41. Narang I, Pike S, Rosenthal M, Balfour-Lynn IM, Bush A. Three-minute step test to assess exercise capacity in children with cystic fibrosis with mild lung disease. Pediatr Pulmonol 2003; 35: 108-113.

42. Brasfield D, Hicks G, Soong S, Peters J, Tiller R. Evaluation of scoring system of the chest radiograph in cystic fibrosis: a collaborative study. Am J Roentgenol 1980; 134: 1195-1198.

43. Munro NC, Han LY, Currie DC, Strickland B, Cole PJ. Radiological evidence of progression of bronchiectasis. Respir Med 1992; 86: 397-401.

44. Nicotra MB. Bronchiectasis. Semin Respir Infect 1994; 9: 3140.

45. Currie DC, Cooke JC, Morgan AD, et al. Interpretation of bronchograms and chest radiographs in patients with chronic sputum production. Thorax 1987; 42: 278-284.

46. Silverman PM, Godwin JD. CT/bronchographic correlations in bronchiectasis. J Comput Assist Tomogr 1987; 11: 52-56.

47. Coates AL, Boyce P, Shaw DG, Godfrey S, Mearns M. Relationship between the chest radiograph, regional lung function studies, exercise tolerance, and clinical condition in cystic fibrosis. Arch Dis Child 1981; 56: 106-111.

48. Hodson CJ, France NE. Pulmonary changes in cystic fibrosis of the pancreas: a radio-pathological study. Clin Radiol 1962; 13: $54-61$.

49. Dagli E. Non cystic fibrosis bronchiectasis. Paediatr Respir Rev 2000; 1: 64-70.

50. Karakoc GB, Yilmaz M, Altintas DU, Kendirli SG. Bronchiectasis: still a problem. Pediatr Pulmonol 2001; 32: 175-178.

51. Robinson T, Leung AN, Northway WH, et al. Composite CT/PFT score: an outcome measure which markedly improves sensitivity to change in early cystic fibrosis lung disease. Pediatr Pulmonol 2002; S24: 298. 